\title{
Transitional Interfaces in Mixed and Cross-Reality: A new frontier?
}

\author{
Hans-Christian Jetter \\ Jan-Henrik Schröder \\ jetter@imis.uni-luebeck.de \\ jschroeder@imis.uni-luebeck.de \\ University of Lübeck \\ Lübeck, Germany \\ Christoph Anthes \\ Christoph.Anthes@fh-hagenberg.at \\ University of Applied Sciences Upper \\ Austria, Hagenberg Campus \\ Hagenberg, Austria
}

\author{
Jan Gugenheimer \\ jan.gugenheimer@telecom-paris.fr \\ Institut Polytechnique de Paris, \\ Télécom Paris \\ Paris, France
}

Mohamed Khamis

Mohamed.Khamis@glasgow.ac.uk

University of Glasgow

Glasgow, United Kingdom

\author{
Mark Billinghurst \\ Mark.Billinghurst@unisa.edu.au \\ University of South Australia \\ Adelaide, Australia \\ University of Auckland \\ Auckland, New Zealand
}

Tiare Feuchtner

tiare.feuchtner@uni-konstanz.de

University of Konstanz

Konstanz, Germany
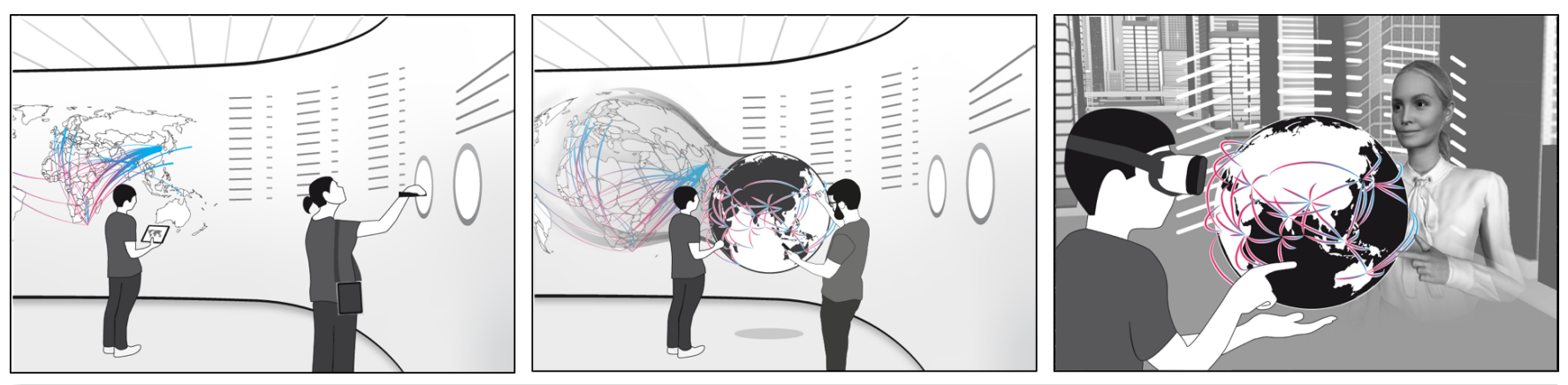

REALITY

VIRTUALITY

Figure 1: Transitional Interfaces (TI) enable users to move between different locations within the reality-virtuality (RV) continuum. They enable users to choose the technology that best supports the task at hand and fulfils their information need.

\begin{abstract}
Transitional interfaces (TIs) and related concepts such as cross-reality (XR) or cross-virtuality (XV) are key topics for future $\mathrm{HCI}$ and $\mathrm{AR} / \mathrm{VR}$ research. Future TIs will enable users to freely move between different locations within the reality-virtuality continuum during work, to choose the best technologies for their task at hand and current information need. Our workshop will explore the core advantages and challenges of TIs and related concepts and address them in presentations and workshop activities at ACM ISS 2021.
\end{abstract}

\section{CCS CONCEPTS}

- Human-centered computing $\rightarrow$ Mixed / augmented reality; Collaborative and social computing devices.

Permission to make digital or hard copies of part or all of this work for personal or classroom use is granted without fee provided that copies are not made or distributed for profit or commercial advantage and that copies bear this notice and the full citation on the first page. Copyrights for third-party components of this work must be honored.

For all other uses, contact the owner/author(s).

ISS '21 Companion, November 14-17, 2021, Lodz, Poland

(c) 2021 Copyright held by the owner/author(s).

ACM ISBN 978-1-4503-8340-0/21/11.

https://doi.org/10.1145/3447932.3487940

\section{KEYWORDS}

transitional interfaces, mixed reality, cross-reality, cross-virtuality, cross-device interaction

\section{ACM Reference Format:}

Hans-Christian Jetter, Jan-Henrik Schröder, Jan Gugenheimer, Mark Billinghurst, Christoph Anthes, Mohamed Khamis, and Tiare Feuchtner. 2021. Transitional Interfaces in Mixed and Cross-Reality: A new frontier? In Interactive Surfaces and Spaces (ISS '21 Companion), November 14-17, 2021, Lodz, Poland. ACM, New York, NY, USA, 4 pages. https://doi.org/10.1145/3447932.3487940

\section{INTRODUCTION}

The rapid proliferation of powerful, yet affordable, off-the-shelf AR/VR head-mounted displays (HMDs) raises questions about how to better integrate such mixed reality technologies into our realworld computing ecosystems and into the real-world physical spaces they inhabit. Currently, application domains for AR/VR technologies such as visual analytics [23, 32, 33], remote assistance [2, 27, 30], training [12, 37, 38], 3D modeling using 2D \& AR simultaneously [31], or prototyping interactive spaces in VR [22] are only beginning to benefit from seamlessly integrating well-established systems 
such as mouse- or keyboard-operated PCs or interactive surfaces like tablets or large touch screens with AR/VR experiences.

We believe that in the future the concept of transitional interfaces (TIs), which was initially introduced in form of the "MagicBook" by Billinghurst et al. in 2001 [4], will provide a key lens through which mixed reality systems should be analysed, understood, and designed to achieve better integration. Billinghurst et al. already envisioned a system that enables a team of users (e.g. an architect and her clients) to move seamlessly between reality and immersive virtual reality while collaborating on the task at hand in the same physical space [4]. In future systems, teams or individuals can seamlessly transition from collaborative 2D interaction with mobile or large screens located in reality (e.g. maps or plots as overviews, Figure 1 left), to also interacting with stereoscopic augmented reality content (e.g. 3D globes as detail view, Figure 1 center), to collaborating in immersive virtual reality systems (e.g. immersive 3D visualization of a location, Figure 1 right).

Grasset et al. formulated theoretical models for TIs in 2006 [14] and also proposed a set of guiding research questions for TIs that remain both relevant and unanswered to this day. In 2012, Carvalho et al. evaluated a TI with regard to its perceptual, functional, and cognitive continuity during transitions in user studies [6]. Still, TIs remain underexplored in $\mathrm{HCI}$ and AR/VR research. Our workshop intends to change this by establishing a dialog in the ACM ISS community and particularly between researchers who are working on TIs or related concepts, such as $\mathrm{XR} / \mathrm{XV}$.

\subsection{Transitional Interfaces - A new frontier?}

We believe that similar to how cross-device interaction [5] has blended tabletop computers, digital whiteboards, and mobile devices with our familiar physical and social environment in meeting rooms [21] or even desk lamps [29], TIs could allow us to seamlessly integrate systems from different locations along the RV-continuum [24] as usable, consistent, and coherent user interfaces in a shared physical space (see Figure 1). This could help achieve "a seamless integration and transition between conventional $2 \mathrm{D}$ visualization, augmented reality and virtual reality in order to provide users with optimal visual and algorithmic support with maximum cognitive and perceptual suitability, depending on their current tasks and needs" [33].

Prior work has explored similar concepts: In their ISS 2020 workshop on XR, Simeone et al. envisioned interfaces that enable "(i) a smooth transition between systems using different degrees of virtuality, or (ii) collaboration between users using different systems with different degrees of virtuality" [35]. We therefore regard XR as closely related to TIs and aim to foster a critical dialog, especially about the definitions and boundaries of XR in comparison to TIs.

In other related work, Reipschläger et al. and Langner et al. use AR to augment interaction and visualization on $2 \mathrm{D}$ interactive surfaces [23, 31, 32]. Guggenheimer et al. bridge the worlds of non-VR and VR users during gaming with a mobile handheld display that serves as a see-through interface into VR [16]. Similar cross-device interaction was later explored by Olin et al. to support collaborative work [26]. At ISMAR 2020, George et al. investigated the design space for seamless, bi-directional transitions along the RVcontinuum [13]. Further, Pointecker et al. explore the audiovisual design and animation of such transitions in their research on $\mathrm{XV}$ analytics [28].

Despite this prior work, we perceive a lack of consistent models, definitions, terminology, and technologies in this space. Therefore, this workshop aims at bringing together researchers who are working on TIs or related concepts such as XR and XV to create a common ground and mutual understanding for developing this space further. We believe that ACM ISS is an optimal venue for such work since it brings together the extensive experience of researchers in the field of interactive surfaces and spaces including cross-device interaction and the $\mathrm{AR} / \mathrm{VR} / \mathrm{XR} / \mathrm{XV}$ community.

\subsection{Focus Topics}

Focus topics of the workshop include but are not limited to:

(1) Defining TIs: Identify differences and commonalities between transitional interfaces and related terms and terminologies (e.g. cross-reality, cross-virtuality, hybrid user interfaces).

(2) Designing TIs: Compare and discuss designs of interaction and visualization techniques that support seamless transitions while maintaining situational and group awareness.

(3) Evaluating TIs: Develop cognitive models or constructs for "seamlessness", "continuity", or other important qualities of TIs and discuss how to "measure" them in user studies and experiments.

(4) Building TIs: Discuss and compare hard- and software that especially supports or hinders seamless transitions, e.g. video-based vs. optical see-through, different AR/VR engines, and platforms for networked virtual environments.

\section{CONCEPT OF THE WORKSHOP}

The workshop will be held as a hybrid, full-day event on Nov. $14^{t h}$, 2021 at ISS 2021 in Łódź. There will be contingency plans for switching to a virtual-only event if the COVID-19 pandemic should not allow for holding a hybrid meeting. Participants who join the workshop only virtually will be able to connect to the workshop room in Łódź using a remote meeting infrastructure, i.e., dedicated cameras/microphones for meetings, Cisco Webex (including installationfree Web clients), Miro for shared whiteboards. This infrastructure will be hosted by the university of the primary organizer and made available free of cost to all workshop participants.

If, in the case of a virtual-only event, the number of participants, their available equipment, and infrastructure should allow for meeting entirely in VR, this will be considered by the organizers. However, we will generally give priority to trusted and stable technologies for collaboration over novel and playful experiences.

\subsection{Before the Workshop}

After acceptance of the workshop, the workshop webpage ${ }^{1}$ will go online and the call for workshop papers will be distributed across relevant $\mathrm{HCI} / \mathrm{AR} / \mathrm{VR}$ mailing lists.

The workshop will be a closed event and participants will need to apply for participation by submitting a 2-4 page position paper by Oct. $18^{t h}$, 2021. Suitable position papers will be selected by the organizers and will be published on the workshop website. There

$\overline{{ }^{1} \text { Workshop website at https://idux-jetter.org/ti-iss21 }}$ 
will be no printed publication of position papers, but a journal theme issue, or special issue, on TIs will be discussed at the workshop.

To keep the submission and selection process as simple and accessible as possible, a standard ACM template will be used and position papers will be collected by e-mail and without the use of a conference system. The selection process will ideally be completed before the early bird registration deadline of ACM ISS 2021.

\subsection{At the Workshop}

The day of the workshop will be divided into four phases:

- Phase 1 (09:00 to 10:00) will be dedicated to invited talks by senior researchers and organizers to establish common ground about the history, terminology, and goals of transitional interfaces and related concepts.

- Phase 2 (10:30 to 12:30) will feature presentations of all submitted position papers.

- Phase 3 (14:00 to 15:30) will involve work in four breakout groups on the four focus topics (see above). However, topics may also be adapted dynamically based on conversations at the workshop.

- Phase 4 (16:00 to 17:00) will be used for breakout groups to report back to the workshop, discuss outcomes, and publication options, and wrapping up the overall event.

\section{ORGANIZERS}

Hans-Christian Jetter is Professor of Interaction Design and User Experience at the University of Lübeck. His research focuses on combining collaborative interactive surfaces and spaces with AR/VR for human-data interaction and information visualization. He has published award-winning papers at CHI, ITS/ISS, and CSCW for the last 10 years [20, 22, 25, 29] and has organized workshops at CHI, AVI, and ITS/ISS.

Jan-Henrik Schröder is $\mathrm{PhD}$ student at the University of Lübeck focusing on collaboration in transitional interfaces.

Jan Gugenheimer is an Assistant Professor of Computer Science at the Institut Polytechnique de Paris (Telecom-Paris), France. In his research, Jan explores upcoming issues that arise when AR/VR technology is starting to be used more frequently outside the users homes. One of the issues he focused on in the past are asymmetric interactions that allow immersed and non-immersed users' to collaborate with each other without the need to have access to the same technology [17-19].

Mark Billinghurst is Director of the Empathic Computing Laboratory, and Professor at the University of South Australia in Adelaide, Australia, and at the University of Auckland, New Zealand. He earned a $\mathrm{PhD}$ in 2002 developing some of the first collaborative $\mathrm{AR}$ systems [3]. Since then he has published over 600 papers on AR, VR, remote collaboration, empathic computing, and related topics. Beginning with MagicBook [4], he has published many papers on transitional Mixed Reality interfaces [15, 36].

Christoph Anthes is Professor of Augmented and Virtual Reality and the head of the Human Interfaces and Virtual Environments (HIVE) group at the University of Applied Sciences Upper Austria. He leads the X-PRO five-year project on cross-virtuality analytics and has published about the intersection of HCI, VR, and visualization $[1,7,11,22]$.
Mohamed Khamis is an assistant professor at the University of Glasgow, UK and has organized workshops on cross-reality interaction [35] and usable security for extended reality[34]. He leads the SIRIUS research group, which has received funding from the UK Research Council, Facebook Reality Labs, the Royal Society of Edinburgh and more.

Tiare Feuchtner is Assistant Professor at the Human-Computer Interaction group at the University of Konstanz. Her main interests are the study of HCI with a focus on embodiment and novel user interfaces for immersive technologies [8-10], as well as multi-user interaction and asymmetric collaboration in AR and VR [26].

\section{ACKNOWLEDGMENTS}

This work is a part of the X-PRO project. The project X-PRO is financed by research subsidies granted by the government of Upper Austria.

\section{REFERENCES}

[1] Christoph Anthes. 2021. X-PRO Project Website. https://x-pro.fh-ooe.at/.

[2] Huidong Bai, Prasanth Sasikumar, Jing Yang, and Mark Billinghurst. 2020. A User Study on Mixed Reality Remote Collaboration with Eye Gaze and Hand Gesture Sharing. In Conference on Human Factors in Computing Systems (CHI). ACM, 1-13. https://doi.org/10.1145/3313831.3376550

[3] Mark Billinghurst. [n.d.]. Shared space: Explorations in collaborative augmented reality [microform]. University of Washington. 345 p. pages.

[4] Mark Billinghurst, Hirokazu Kato, and Ivan Poupyrev. 2001. The MagicBook moving seamlessly between reality and virtuality. IEEE Computer Graphics and Applications 21, 3 (2001), 6-8. https://doi.org/10.1109/38.920621

[5] Frederik Brudy, Christian Holz, Roman Rädle, Chi-Jui Wu, Steven Houben, Clemens Nylandsted Klokmose, and Nicolai Marquardt. 2019. Cross-Device Taxonomy: Survey, Opportunities and Challenges of Interactions Spanning Across Multiple Devices. In Proceedings of the 2019 CHI Conference on Human Factors in Computing Systems. Association for Computing Machinery, New York, NY, USA, 1-28. https://doi.org/10.1145/3290605.3300792

[6] Felipe G. Carvalho, Daniela G. Trevisan, and Alberto Raposo. 2012. Toward the Design of Transitional Interfaces: An Exploratory Study on a Semi-Immersive Hybrid User Interface. Virtual Real. 16, 4 (Nov. 2012), 271-288. https://doi.org/ 10.1007/s10055-011-0205-y

[7] Barrett Ens, Benjamin Bach, Maxime Cordeil, Ulrich Engelke, Marcos Serrano, Wesley Willett, Arnaud Prouzeau, Christoph Anthes, Wolfgang Büschel, Cody Dunne, Tim Dwyer, Jens Grubert, Jason H. Haga, Nurit Kirschenbaum, Dylan Kobayashi, Tica Lin, Monsurat Olaosebikan, Fabian Pointecker, David Saffo, Nazmus Saquib, Dieter Schmalsteig, Danielle Albers Szafir, Matthew Whitlock, and Yalong Yang. 2021. Grand Challenges in Immersive Analytics. In Conference on Human Factors in Computing Systems (CHI). ACM. https://doi.org/10.1145/ 3411764.3446866

[8] João Marcelo Evangelista Belo, Anna Maria Feit, Tiare Feuchtner, and Kaj Grønbæk. 2021. XRgonomics: Facilitating the Creation of Ergonomic 3D Interfaces. In Proceedings of the 2021 CHI Conference on Human Factors in Computing Systems. Association for Computing Machinery, New York, NY, USA, Article 290, 11 pages. https://doi.org/10.1145/3411764.3445349

[9] Tiare Feuchtner and Jörg Müller. 2017. Extending the Body for Interaction with Reality. In Proceedings of the 2017 CHI Conference on Human Factors in Computing Systems. Association for Computing Machinery, New York, NY, USA, 5145-5157. https://doi.org/10.1145/3025453.3025689

[10] Tiare Feuchtner and Jörg Müller. 2018. Ownershift: Facilitating Overhead Interaction in Virtual Reality with an Ownership-Preserving Hand Space Shift. In Proceedings of the 31st Annual ACM Symposium on User Interface Software and Technology (Berlin, Germany) (UIST '18). Association for Computing Machinery, New York, NY, USA, 31-43. https://doi.org/10.1145/3242587.3242594

[11] Rubén Jesús García-Hernández, Christoph Anthes, Markus Wiedemann, and Dieter Kranzlmüller. 2016. Perspectives for Using Virtual Reality to Extend Visual Data Mining in Information Visualization. In Aerospace Conference. IEEE, 1-11. https://doi.org/10.1109/AERO.2016.7500608

[12] Nirit Gavish, Teresa Gutiérrez, Sabine Webel, Jorge Rodríguez, Matteo Peveri, Uli Bockholt, and Franco Tecchia. 2015. Evaluating virtual reality and augmented reality training for industrial maintenance and assembly tasks. Interactive Learning Environments 23, 6 (2015), 778-798.

[13] Ceenu George, An Ngo Tien, and Heinrich Hussmann. 2020. Seamless, Bidirectional Transitions along the Reality-Virtuality Continuum: A Conceptualization and Prototype Exploration. In International Symposium on Mixed and 
Augmented Reality (ISMAR). IEEE, 412-424. https://doi.org/10.1109/ISMAR50242. 2020.00067

[14] Raphael Grasset, Julian Looser, and Mark Billinghurst. 2006. Transitional Interface: Concept, Issues and Framework. In International Symposium on Mixed and Augmented Reality (ISMAR). IEEE. https://doi.org/10.1109/ismar.2006.297819

[15] Raphael Grasset, Alessandro Mulloni, Mark Billinghurst, and Dieter Schmalstieg. 2011. Navigation Techniques in Augmented and Mixed Reality: Crossing the Virtuality Continuum. Springer New York, New York, NY, 379-407. https://doi.org/10.1007/978-1-4614-0064-6_18

[16] Jan Gugenheimer, Evgeny Stemasov, Julian Frommel, and Enrico Rukzio. 2017 ShareVR: Enabling Co-Located Experiences for Virtual Reality between HMD and Non-HMD Users. In Conference on Human Factors in Computing Systems (CHI). ACM, 4021--4033. https://doi.org/10.1145/3025453.3025683

[17] Jan Gugenheimer, Evgeny Stemasov, Julian Frommel, and Enrico Rukzio. 2017 ShareVR: Enabling Co-Located Experiences for Virtual Reality between HMD and Non-HMD Users. In Proceedings of the 2017 CHI Conference on Human Factors in Computing Systems. Association for Computing Machinery, New York, NY, USA, 4021-4033. https://doi.org/10.1145/3025453.3025683

[18] Jan Gugenheimer, Evgeny Stemasov, Harpreet Sareen, and Enrico Rukzio. 2018 FaceDisplay: Towards Asymmetric Multi-User Interaction for Nomadic Virtual Reality. Association for Computing Machinery, New York, NY, USA, 1-13. https: //doi.org/10.1145/3173574.3173628

[19] Pascal Jansen, Fabian Fischbach, Jan Gugenheimer, Evgeny Stemasov, Julian Frommel, and Enrico Rukzio. 2020. ShARe: Enabling Co-Located Asymmetric Multi-User Interaction for Augmented Reality Head-Mounted Displays. In Proceedings of the 33rd Annual ACM Symposium on User Interface Software and Technology (Virtual Event, USA) (UIST '20). Association for Computing Machinery, New York, NY, USA, 459-471. https://doi.org/10.1145/3379337.3415843

[20] Hans-Christian Jetter, Jens Gerken, Michael Zöllner, Harald Reiterer, and Natas Milic-Frayling. 2011. Materializing the Query with Facet-Streams: A Hybrid Surface for Collaborative Search on Tabletops. In Conference on Human Factors in Computing Systems (CHI). ACM, 3013-3022. https://doi.org/10.1145/1978942. 1979390

[21] Hans-Christian Jetter, Harald Reiterer, and Florian Geyer. 2014. Blended Interaction: Understanding Natural Human-Computer Interaction in Post-WIMP Interactive Spaces. Personal and Ubiquitous Computing 18, 5 (2014), 1139-1158. https://doi.org/10.1007/s00779-013-0725-4

[22] Hans-Christian Jetter, Roman Rädle, Tiare Feuchtner, Christoph Anthes, Judith Friedl, and Clemens Nylandsted Klokmose. 2020. "In VR, Everything Is Possible!": Sketching and Simulating Spatially-Aware Interactive Spaces in Virtual Reality. In Conference on Human Factors in Computing Systems (CHI). ACM, 1-16. https: //doi.org/10.1145/3313831.3376652

[23] Ricardo Langner, Marc Satkowski, Wolfgang Büschel, and Raimund Dachselt. 2021. MARVIS: Combining Mobile Devices and Augmented Reality for Visual Data Analysis. In Proceedings of the 2021 ACM Conference on Human Factors in Computing Systems (Yokohama, Japan). ACM, New York, NY, USA, 17 pages. https://doi.org/10.1145/3411764.3445593

[24] Paul Milgram and Fumio Kishino. 1994. A Taxonomy of Mixed Reality Visual Displays. IEICE Transactions on Information and Systems E77-D, 12 (1994), 13211329.

[25] Thomas Neumayr, Hans-Christian Jetter, Mirjam Augstein, Judith Friedl, and Thomas Luger. 2018. Domino: A Descriptive Framework for Hybrid Collaboration and Coupling Styles in Partially Distributed Teams. Proceedings of the ACM on Human-Computer Interaction 2, CSCW, Article 128 (2018), 24 pages. https: //doi.org/10.1145/3274397

[26] Patrick Aggergaard Olin, Ahmad Mohammad Issa, Tiare Feuchtner, and Kaj Grøn bæk. 2020. Designing for Heterogeneous Cross-Device Collaboration and Social Interaction in Virtual Reality. In 32nd Australian Conference on Human-Computer Interaction (Sydney, NSW, Australia) (OzCHI '20). Association for Computing Machinery, New York, NY, USA, 112-127. https://doi.org/10.1145/3441000.3441070

[27] Thammathip Piumsomboon, Youngho Lee, Gun Lee, and Mark Billinghurst. 2017 CoVAR: A Collaborative Virtual and Augmented Reality System for Remote Collaboration. In SIGGRAPH Asia (SA) - Emerging Technologies. ACM, 1-2. https: //doi.org/10.1145/3132818.3132822

[28] Fabian Pointecker, Hans-Christian Jetter, and Christoph Anthes. 2020. Exploration of Visual Transitions Between Virtual and Augmented Reality. In Workshop on Immersive Analytics: Envisioning Future Productivity for Immersive Analytics // @CHI 2020 Honolulu.

[29] Roman Rädle, Hans-Christian Jetter, Nicolai Marquardt, Harald Reiterer, and Yvonne Rogers. 2014. HuddleLamp: Spatially-Aware Mobile Displays for Ad-Hoc Around-the-Table Collaboration. In Proceedings of the Ninth ACM International Conference on Interactive Tabletops and Surfaces (Dresden, Germany) (ITS '14). Association for Computing Machinery, New York, NY, USA, 45-54. https://doi. org $/ 10.1145 / 2669485.2669500$

[30] Troels Ammitsbøl Rasmussen and Kaj Gronbak. 2019. Tailorable Remote Assistance with RemoteAssistKit: A Study of and Design Response to Remote Assistance in the Manufacturing Industry. In International Conference on Collaboration and Technology. Springer, 80-95.
[31] Patrick Reipschläger, Severin Engert, and Raimund Dachselt. 2020. Augmented Displays: Seamlessly Extending Interactive Surfaces with Head-Mounted Augmented Reality. In Proceedings of the 2020 CHI Conference Extended Abstracts on Human Factors in Computing Systems (Honolulu, Hawaii, USA). ACM, New York, 4 pages. https://doi.org/10.1145/3334480.3383138

[32] Patrick Reipschläger, Tamara Flemisch, and Raimund Dachselt. 2021. Personal Augmented Reality for Information Visualization on Large Interactive Displays. IEEE Transactions on Visualization and Computer Graphics 27 (2 2021), 1182-1192. Issue 2. https://doi.org/10.1109/TVCG.2020.3030460

[33] Andreas Riegler, Christoph Anthes, Hans-Christian Jetter, Christoph Heinzl, Clemens Holzmann, Herbert Jodlbauer, Manuel Brunner, Stefan Auer, Judith Friedl, Bernhard Fröhler, Christina Leitner, Fabian Pointecker, Daniel Schajda, and Shailesh Tripathi. 2020. Cross-Virtuality Visualization, Interaction and Collaboration. In International Workshop on Cross-Reality (XR) Interaction @ ACM ISS 2020. http://ceur-ws.org/Vol-2779/paper1.pdf

[34] Stefan Schneegass, Mohamed Khamis, Florian Alt, Uwe Gruenefeld, Karola Marky, Alia Saad, Jonathan Liebers, Jonas Auda, Florian Mathis, and Lukas Mecke. 2021. VR4Sec Security for XR and XR for Security - Workshop Website. https://vr4sec. hcigroup.de/index.php.

[35] Adalberto L. Simeone, Mohamed Khamis, Augusto Esteves, Florian Daiber, Matjaž Kljun, Klen Čopič Pucihar, Poika Isokoski, and Jan Gugenheimer. 2020. International Workshop on Cross-Reality (XR) Interaction. In Companion Proceedings of the 2020 Conference on Interactive Surfaces and Spaces (Virtual Event, Portugal) (ISS '20). Association for Computing Machinery, New York, NY, USA, 111-114. https://doi.org/10.1145/3380867.3424551

[36] Theophilus Teo, Louise Lawrence, Gun A. Lee, Mark Billinghurst, and Matt Adcock. 2019. Mixed Reality Remote Collaboration Combining 360 Video and 3D Reconstruction. In Proceedings of the 2019 CHI Conference on Human Factors in Computing Systems. Association for Computing Machinery, New York, NY, USA, 1-14. https://doi.org/10.1145/3290605.3300431

[37] Sabine Webel, Uli Bockholt, Timo Engelke, Matteo Peveri, Manuel Olbrich, and Carsten Preusche. 2011. Augmented reality training for assembly and maintenance skills. In BIO web of conferences, Vol. 1. EDP Sciences, 00097.

[38] Frederik Winther, Linoj Ravindran, Kasper Paabøl Svendsen, and Tiare Feuchtner. 2020. Design and evaluation of a vr training simulation for pump maintenance based on a use case at grundfos. In 2020 IEEE Conference on Virtual Reality and $3 D$ User Interfaces (VR). IEEE, 738-746. 